\title{
RACK1 expression contributes to JNK activity, but JNK activity does not enhance RACK1 expression in hepatocellular carcinoma SMMC-7721 cells
}

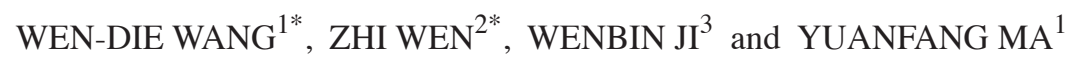 \\ ${ }^{1}$ Key Laboratory of Cellular and Molecular Immunology, Henan University, Kaifeng, Henan 475001; \\ ${ }^{2}$ Division of Internal Medicine and ${ }^{3}$ Department of Hepatobiliary Surgery, \\ The Chinese PLA General Hospital, Beijing 100853, P.R. China
}

Received February 9, 2014; Accepted November 25, 2014

DOI: $10.3892 / \mathrm{ol} .2015 .3129$

\begin{abstract}
Receptor for activated C kinase 1 (RACK1) is up-regulated in hepatocellular carcinoma (HCC) and has been reported to augment c-Jun $\mathrm{N}$-terminal protein kinase (JNK) activity in HCC SMMC-7721 cells. By contrast, activator protein-1, a downstream JNK transcription factor, has been revealed to mediate the overexpression of RACK1 in melanoma cells. Therefore, the association between RACK1 and JNK activity in HCC cells has yet to be completely elucidated. The present study analyzed the effects of RACK1 or JNK loss of function on the levels of RACK1 protein, JNK activity, cell proliferation and apoptosis induced by tumor necrosis factor-related apoptosis inducing ligand in HCC SMMC-7721 cells. It was found that JNK loss of function exhibited no effect on RACK1 expression, whereas a loss of RACK1 function led to reduced JNK activity in SMMC-7721 cells. RACK1 and JNK loss of function resulted in the impaired oncogenic growth of SMMC-7721 cells. The present data further support a pivotal role of RACK1 in mediating enhanced JNK activity in HCC cells and also indicate that a novel mechanism exists for RACK1 overexpression in HCC SMMC-7721 cells.
\end{abstract}

Correspondence to: Professor Yuanfang Ma, Key Laboratory of Cellular and Molecular Immunology, Henan University, 300 Jin-Ming Da dao, Kaifeng, Henan 475001, P.R. China

E-mail: mayf@henu.edu.cn

Dr Wenbin Ji, Department of Hepatobiliary Surgery, The Chinese PLA General Hospital, 28 Fuxing Street, Beijing 100853, P.R. China E-mail: jiwenbin1999@126.com

*Contributed equally

Key words: Receptor for activated C kinase 1, mitogen-activated protein kinase kinase 7, c-Jun $\mathrm{N}$-terminal protein kinase, hepatocellular carcinoma

\section{Introduction}

Hepatocellular carcinoma (HCC) is one of the most common and lethal cancers in the human population, ranked the third most common cause of cancer-associated mortality worldwide, particularly in Africa and Asia (1). Although persistent viral infections and persistent exposure to hepatotoxic agents play a role in HCC neoplastic transformation (1), the underlying mechanism controlling the development and progression of $\mathrm{HCC}$ is largely unclear.

Recently, c-Jun N-terminal protein kinase (JNK) has been reported to be involved in regulating liver tumorigenesis. JNK belongs to the mitogen-activated protein kinase (MAPK) superfamily, which also includes extracellular signal-regulated kinase (ERK) and the p38 family of kinases (2-5). The activation of JNK is mediated by sequential protein phosphorylation through a MAPK module. MAPK kinase (MKK) 7 and MKK4 play a non-redundant role in the dual phosphorylation of JNK at Thr183 and Tyr185, which is required for JNK activity (2-5). Once activated, JNK phosphorylates and activates c-Jun, a key component of the transcription factor activator protein-1 (AP-1) (2-5). Elevated levels of JNK activity have been frequently observed in HCC and have been demonstrated to contribute to HCC growth by promoting cell proliferation and resistance to tumor necrosis factor-related apoptosis inducing ligand (TRAIL)-mediated apoptosis (2-5).

Receptor for activated $\mathrm{C}$ kinase 1 (RACK1), coded for by the GNB2L1 gene, is a scaffold protein with a propeller-like structure of seven WD40 repeats (6-10). Numerous studies have suggested that RACK1 plays a pivotal role in the coordination of cell growth, migration and differentiation during tumorigenesis (6-10). It has been demonstrated that RACK1 is up-regulated in $\mathrm{HCC}$ and that overexpressed RACK1 augments JNK activity, thereby promoting HCC growth by directly binding to MKK7 and enhancing MKK7 activity (11).

It has been reported that there is an AP-1 site in the promoter region of the GNB2L1 gene (12). In addition, AP-1 has been revealed to mediate RACK1 overexpression in melanoma cells (13). Since enhanced JNK activity can lead to elevated AP-1 activity in various cell contexts (14), it is 
important to investigate the association between RACK1 and the JNK pathway in HCC SMMC-7721 cells. The aim of the present study was to determine whether JNK activity regulates RACK1 expression and whether RACK1 regulates JNK activity in HCC SMMC-7721 cells.

\section{Materials and methods}

Cell culture and transduction. HCC SMMC-7721 cells were purchased from the Shanghai Institutes for Biological Sciences (Shanghai, China) and were cultured in Dulbecco's modified Eagle's medium supplemented with $10 \%$ fetal bovine serum, 100 units $/ \mathrm{ml}$ penicillin and $100 \mu \mathrm{g} / \mathrm{ml}$ streptomycin, and were maintained at $37^{\circ} \mathrm{C}$ in a $5 \% \mathrm{CO}_{2}$ atmosphere. Lentivirus-based RACK1 short hairpin (sh)RNA, 5'-GGATGAGACCAACTA TGGA-3', JNK shRNA, 5'-AAAGAAUGUCCUACCUUCU-3', and control lentivirus were obtained from Shanghai GeneChem Co., Ltd. (Shanghai, China). Transduction was performed using lentivirus, at a multiplicity of infection of 10 .

Immunoblotting analysis. The SMMC-7721 cells were washed twice with ice-cold phosphate-buffered saline (PBS) and were then lysed using $20 \mathrm{mM}$ Tris/ $\mathrm{HCl}$ (pH 7.6), $250 \mathrm{mM} \mathrm{NaCl}$, $3 \mathrm{mM}$ EDTA, $3 \mathrm{mM}$ EGTA, 0.5\% NP40, $1 \mathrm{mM}$ dithiothreitol, $5 \mathrm{mM} \mathrm{NaF}, 2 \mathrm{mM} \mathrm{Na}_{3} \mathrm{VO}_{4}$ and $0.2 \mu \mathrm{M}$ aprotinin. The whole cell extract was clarified at $10,000 \mathrm{x}$ g for $15 \mathrm{~min}$ at $4^{\circ} \mathrm{C}$. The recovered protein was quantified using a Bradford protein assay. Equal quantities of proteins were resolved by sodium dodecyl sulfate-polyacrylamide gel electrophoresis (SDS-PAGE) and the proteins were then transferred to Hybond-P polyvinylidene difluoride (PVDF) membranes (GE Healthcare Life Sciences, Chalfont, UK). The membranes were initially incubated with primary antibody over night at $4^{\circ} \mathrm{C}$, and then with horseradish peroxidase-conjugated polyclonal goat anti-rabbit or anti-mouse secondary antibodies (cat. no. ZB2301 and ZB2305, respectively; 1:5,000; Beijing Zhongshan Golden Bridge Biotechnology Co., Ltd., Beijing, China) for $1 \mathrm{~h}$ at room temperature. Bound antibody was detected using an electrochemiluminescence kit (Amersham, Chalfont, UK) and Kodak X-ray film (Rochester, NY, USA). Rabbit anti-human polyclonal antibodies against MKK7 (cat. no. 4172; 1:1,000), phosphorylated MKK7 (P-MKK7; cat. no. 4171; 1:1,000), and phosphorylated JNK (P-JNK; cat. no. 9251; 1:1,000) were purchased from Cell Signaling Technology, Inc. (Danvers, MA, USA). Monoclonal mouse anti-human antibodies against RACK1 (cat. no 610171; $1: 5,000$ ) and JNK (cat. no. 612541; 1:1,000) were obtained from BD Biosciences (Franklin Lakes, NJ, USA). Monoclonal mouse anti-human antibody against $\beta$-actin (cat. no. sc-8432; 1:5,000) was obtained from Santa Cruz Biotechnology, Inc. (Dallas, TX, USA). All the chemical inhibitors were purchased from Calbiochem (Billerica, MA, USA).

Soft-agar assays. Agar (1.2\%) was mixed with 2X Dulbecco's modified Eagle's medium at a ratio of 1:1 to produce a $0.6 \%$ agar growth medium solution. Next, $1.5 \mathrm{ml}$ of the $0.6 \%$ growth medium mixture was pipetted into each well of a six-well cell culture cluster (Corning Life Sciences, Corning, New York, NY, USA), while avoiding bubble formation. The mixture was then evenly spread by slowly rotating the plate. The $0.6 \%$ agar growth medium layer was left to harden for $20 \mathrm{~min}$ at $4^{\circ} \mathrm{C}$

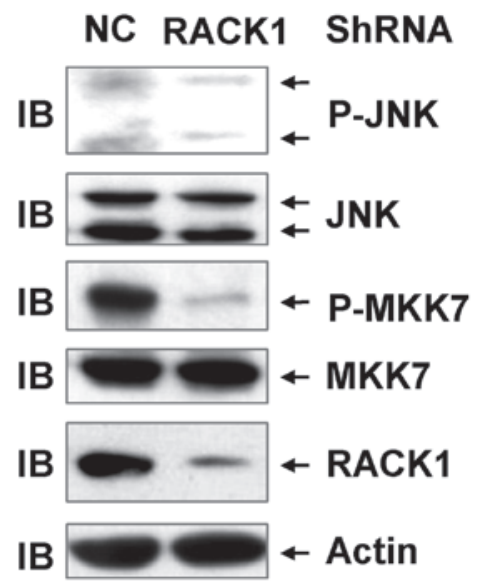

Figure 1. RACK1 knockdown leads to impaired MKK7/JNK activation in SMMC-7721 cells. Cell lysates were harvested and subjected to IB with the indicated antibodies $96 \mathrm{~h}$ after infection of the SMMC7721 cells with control lentivirus or lentivirus carrying RACK1 shRNA. NC, negative control; RACK1, receptor for activated C kinase 1; shRNA, short hairpin RNA; JNK, c-Jun N-terminal protein kinase; P-JNK, phosphorylated JNK; MKK7, mitogen-activated protein kinase kinase 7; P-MKK7, phosphorylated MKK7; IB, immunoblotting.

and the cells were then seeded at a density of $1 \times 10^{3}$ cells $/ \mathrm{ml}$ in $0.3 \%$ agar diluted with $2 \mathrm{X}$ Dulbecco's modified Eagle's medium, at a ratio of 1:1. Cell suspension $(1 \mathrm{ml})$ was plated onto the $0.6 \%$ agar growth medium plate and cultured at $37^{\circ} \mathrm{C}$ in a $5 \% \mathrm{CO}_{2}$ atmosphere for 14 days. The colony numbers were counted using a microscope (Nikon Eclipse TS100; Nikon Corporation, Tokyo, Japan), based on colonies $>400 \mu \mathrm{m}$ in diameter.

Apoptosis analysis. The cells were adjusted to a density of $2 \times 10^{5}$ cells $/ \mathrm{ml}$ and were added to 24 -well plates, with $0.5 \mathrm{ml}$ in each well. TRAIL was purchased from Sigma-Aldrich (St. Louis, MO, USA) and was used to treat the SMMC-7721 cells according to the manufacturer's instructions. The cells were washed with PBS twice and stained with Annexin V-phycoerythrin and 7-aminoactinomycin D (Nanjing KeyGen Biotech, Nanjing, Jiangsu, China) for $15 \mathrm{~min}$ at room temperature in the dark. The level of apoptosis was determined by measuring the fluorescence of the cells using a flow cytometer (BD Biosciences).

Statistical analysis. Statistically significant differences between groups were identified using a two-tailed Student's $\mathrm{t}$-test. $\mathrm{P}<0.05$ was considered to indicate a statistically significant result.

\section{Results}

Enhancement of MKK7/JNK activity by RACK1 in SMMC-7721 cells. To investigate the correlation between RACK1 and the JNK pathway in SMMC-7721 cells, endogenous RACK1 expression was transiently silenced using lentivirus-based RACK1 shRNA. The expression of RACK1 protein was detected by immunoblotting analysis with an antibody against RACK1, whereas JNK activity and MKK7 activity was measured by immunoblotting analysis of 


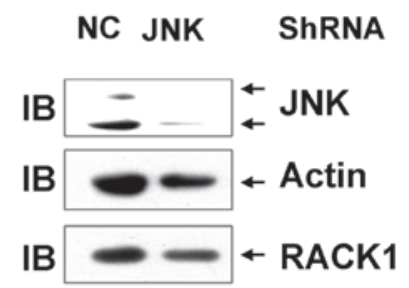

Figure 2. JNK knockdown exhibits no effect on RACK1 expression. Cell lysates were harvested and subjected to IB with the indicated antibodies $96 \mathrm{~h}$ after infection of SMMC-7721 cells with control lentivirus or lentivirus carrying JNK shRNA. NC, negative control; JNK, c-Jun N-terminal protein kinase; shRNA, short hairpin RNA; RACK1, receptor of activated C kinase 1 ; IB, immunoblotting.

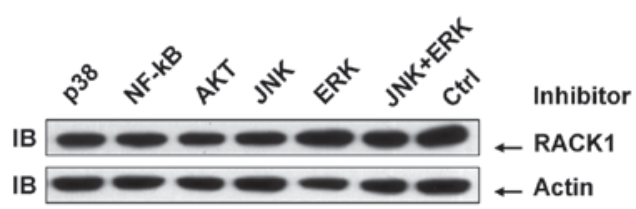

Figure 3. JNK inhibitor exhibits no effect on RACK1 expression. SMMC7721 cells were exposed to treatment with the indicated inhibitors for $48 \mathrm{~h}$. Cell lysates were then harvested and subjected to IB with the indicated antibodies. The final concentrations of the p38 inhibitor SB203580, NF- $\mathrm{KB}$ inhibitor Bay11-7082, AKT inhibitor wortmannin, JNK inhibitor SP600125 or ERK inhibitor U0126 were $10 \mu \mathrm{M}$. IB, immunoblotting; NF-kB, nuclear factor $\kappa \mathrm{B}$; JNK, c-Jun $\mathrm{N}$-terminal protein kinase; AKT, protein kinase B; ERK, extracellular signal-related kinase; cntrl, control.

A

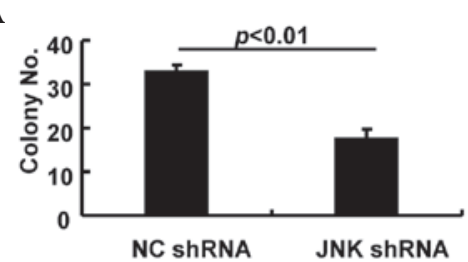

B

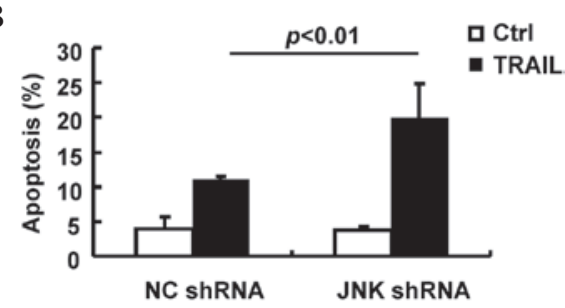

Figure 4. JNK knockdown led to reduced anchorage-independent growth and enhanced sensivity to TRAIL-induced cell death. The cells were plated in soft agar $\left(1 \times 10^{3} /\right.$ well $) 96 \mathrm{~h}$ after infection of SMMC-7721 cells with control lentivirus or lentivirus carrying JNK shRNA, and (A) were assayed to determine the colony number after 14 days, or (B) the cells were treated with $100 \mathrm{ng} / \mathrm{ml}$ TRAIL for $24 \mathrm{~h}$, followed by Annexin V staining. The values reported are the mean \pm standard deviation. No., number; $\mathrm{NC}$, negative control; shRNA, short hairpin RNA; JNK, c-Jun N-terminal protein kinase; Ctrl, control; TRAIL, tumour necrosis factor-related apoptosis inducing ligand.

P-JNK and P-MKK7, respectively. The present results demonstrate that transduction of SMMC-7721 cells with the lentivirus carrying RACK1 shRNA compared with the control lentivirus, significantly decreased RACK1 expression (Fig. 1). Silencing of endogenous RACK1 expression by RACK1 shRNA in SMMC-7721 cells significantly suppressed the basal level of
A

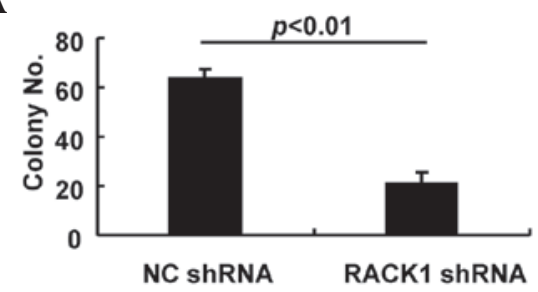

$\mathbf{B}$

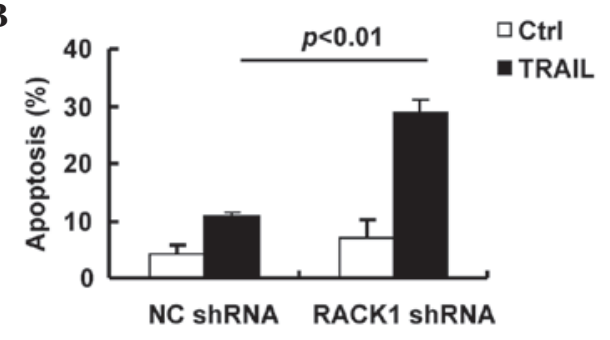

Figure 5. RACK1 knockdown led to reduced anchorage-independent growth and enhanced sensivity to TRAIL-induced cell death. The cells were plated in soft agar (1x10 $/$ well) $96 \mathrm{~h}$ after infection of SMMC-7721 cells with control lentivirus or lentivirus carrying RACK1 shRNA, and (A) were assayed to determine the colony number after 14 days, or (B) the cells were treated with $100 \mathrm{ng} / \mathrm{ml}$ TRAIL for $24 \mathrm{~h}$, followed by Annexin V staining. The values reported are the mean \pm standard deviation. No., number; NC, negative control; shRNA, short hairpin RNA; RACK1, receptor for activated C kinase 1; Ctrl, control; TRAIL, tumour necrosis factor-related apoptosis inducing ligand.

P-JNK (Fig. 1). In addition, the present data also revealed that a decreased level of P-JNK in RACK1-knockdown cells was associated with a reduced P-MKK7 level (Fig. 1). These data collectively indicate that RACK1 contributes to the enhancement of MKK7/JNK activity in SMMC-7721 cells.

JNK activity plays no role in RACK1 overexpression in SMMC-7721 cells. Endogenous JNK expression was transiently silenced using lentivirus-based JNK shRNA. The present results demonstrate that transduction of SMMC-7721 cells with the JNK shRNA-carrying lentivirus significantly decreased the expression of the p54 JNK and p46 JNK proteins compared to transfection with the control lentivirus (Fig. 2). However, the protein level of RACK1 was not reduced when JNK was knocked down (Fig. 2). These data suggest that JNK activity does not contribute to RACK1 overexpression in SMMC-7721 cells.

As AP-1 activity is affected by other MAPK superfamily members in addition to JNK (14), chemically synthesized inhibitors were used to block the activity of ERK, p38 and JNK. Akt and NF- $\mathrm{KB}$ inhibitors were also included. Treatment of SMMC-7721 cells with these inhibitors for $48 \mathrm{~h}$ exhibited no significant effects on the RACK protein level (Fig. 3). These data further confirm that JNK activity plays no role in RACK1 overexpression in SMMC-7721 cells. However, these data also suggest that other factors, but not AP-1, mediate RACK1 overexpression in SMMC-7721 cells.

JNK activity contributes to the oncogenic growth of SMMC-7721 cells. It is of importance to clarify the roles of JNK activity in the tumorigenic growth of SMMC-7721 cells. In this scenario, anchorage-independent growth and apoptosis in response to TRAIL were analyzed in SMMC-7721 cells 
transduced with the control lentivirus or the lentivirus carrying JNK shRNA. The present data reveal that transduction of SMMC-7721 cells with the lentivirus carrying JNK shRNA significantly inhibited the anchorage-independent growth compared with the control lentivirus (Fig. 4A) and led to an increased proportion of apoptotic cells in response to TRAIL (Fig. 4B). Thus, JNK activity makes an essential contribution to the oncogenic growth of SMMC-7721 cells.

RACK1 facilitates the oncogenic growth of SMMC-7721 cells. The previous data suggested that JNK activity contributes to the oncogenic growth of SMMC-7721 cells by promoting cell proliferation and resistance to TRAIL-mediated apoptosis. Since RACK1 enhances MKK7/JNK activity in this cell line, it is important to investigate how RACK1 may affect tumorigenic growth. Therefore, anchorage-independent growth and apoptosis in response to TRAIL were analyzed in SMMC-7721 cells transduced with the control lentivirus or the lentivirus carrying RACK1 shRNA. As expected, transduction of SMMC-7721 cells with the lentivirus carrying RACK1 shRNA significantly inhibited anchorage-independent growth compared with the control lentivirus (Fig. 5A) and led to increased apoptosis in response to TRAIL (Fig. 5B). Thus, RACK1 facilitates the oncogenic growth of SMMC-7721 cells, partially due to the enhancement of JNK activity.

\section{Discussion}

It has been reported that RACK1 promotes HCC growth by enhancing MKK7 activity (11). The correlation between the levels of RACK1 protein and the activity of the JNK pathway was observed in clinical HCC tissues and various HCC cell lines (11). However, it should be noted that SMMC-7721, BEL-7402 and BEL-7404 cells exhibit significantly elevated RACK1 expression, but the levels of P-JNK in these cells are only weakly up-regulated (11). This issue promoted an examination of the association between RACK1 and JNK in SMMC-7721 cells. The present data provided additional support for a pivotal role of RACK1 in mediating enhanced JNK activity and in HCC growth.

The present data revealed that the JNK activity, although weakly detected, is essential to the oncogenic growth of SMMC-7721 cells. Consistent with the present observations, a recent study indicates that eupolyphaga sinensis walker extract (ESWE) demonstrated significant inhibition on the growth of SMMC-7721 cells, which was associated with decreased JNK1 protein expression (15). Silencing of endogenous RACK1 expression leads to reduced JNK activity and impaired oncogenic growth of SMMC-7721 cells. Thus, RACK1 promotes the tumorigenic growth of SMMC-7721 cells through an at least partial enhancement of JNK activity, although the role of RACK1 on the activity of the JNK pathway may be compromised by other genetic mutations in SMMC-7721 cells. Further explorations are required to clarify the genetic mutations that compromise JNK activity and the consequent effects in HCC development and progression.

The molecular mechanisms underlying the elevated RACK1 expression are undefined. It has been reported that there is an AP-1 site in the promoter region of the GNB2L1 gene (12). Additionally, AP-1 has been revealed to mediate RACK1 overexpression in melanoma cells (13). Since enhanced JNK activity can lead to elevated AP-1 activity in various cell contexts (14), the present study detected RACK1 protein levels subsequent to knockdown of JNK or inhibition of JNK activity by a chemical inhibitor. The data revealed that expression of RACK1 is independent of the activation of JNK signaling. As p38 and ERK also contribute to AP-1 activity (14), and it has been reported that a NF- $\mathrm{KB}$ site in the promoter region of the GNB2L1 gene mediates up-regulation of RACK1 $(16,17)$, chemical inhibitors of p38, ERK, NF- $\mathrm{kB}$ and Akt were also investigated in the present study. Furthermore, neither the blockade of JNK signaling nor a blockade of $\mathrm{p} 38$, ERK, AKT or NF- $\mathrm{kB}$ signaling for $48 \mathrm{~h}$ demonstrated any effect on the RACK1 protein level. Therefore, there may be alternative mechanisms underlying RACK1 upregulation.

\section{References}

1. Llovet JM, Burroughs A and Bruix J: Hepatocellular carcinoma. Lancet 362: 1907-1917, 2003.

2. Hui L, Zatloukal K, Scheuch H, et al: Proliferation of human HCC cells and chemically induced mouse liver cancers requires JNK1-dependent p21 downregulation. J Clin Invest 118: 3943-3953, 2008.

3. Sakurai T, Maeda S, Chang L and Karin M: Loss of hepatic NF-kappa B activity enhances chemical hepatocarcinogenesis through sustained c-Jun $\mathrm{N}$-terminal kinase 1 activation. Proc Natl Acad Sci USA 103: 10544-10551, 2006.

4. Mucha SR, Rizzani A, Gerbes AL, et al: JNK inhibition sensitises hepatocellular carcinoma cells but not normal hepatocytes to the TNF-related apoptosis-inducing ligand. Gut 58: 688-698, 2009.

5. Kuntzen C, Sonuc N, De Toni EN, et al: Inhibition of c-Jun-N-terminal-kinase sensitizes tumor cells to CD95-induced apoptosis and induces $\mathrm{G} 2 / \mathrm{M}$ cell cycle arrest. Cancer Res 65: 6780-6788, 2005.

6. Hermanto U, Zong CS, Li W and Wang LH: RACK1, an insulin-like growth factor I (IGF-I) receptor-interacting protein, modulates IGF-I-dependent integrin signaling and promotes cell spreading and contact with extracellular matrix. Mol Cell Biol 22: 2345-2365, 2002.

7. Kadrmas JL, Smith MA, Pronovost SM and Beckerle MC: Characterization of RACK1 function in Drosophila development. Dev Dyn 236: 2207-2215, 2007.

8. Hoffmann B, Wanke C, Lapaglia SK and Braus GH: c-Jun and RACK1 homologues regulate a control point for sexual development in Aspergillus nidulans. Mol Microbiol 37: 28-41, 2000.

9. McLeod M, Shor B, Caporaso A, et al: Cpc2, a fission yeast homologue of mammalian RACK1 protein, interacts with Ran1 (Pat1) kinase to regulate cell cycle progression and meiotic development. Mol Cell Biol 20: 4016-4027, 2000.

10. Rothberg KG, Burdette DL, Pfannstiel J, et al: The RACK1 homologue from Trypanosoma brucei is required for the onset and progression of cytokinesis. J Biol Chem 281: 9781-9790, 2006.

11. Guo Y, Wang W, Wang J, et al: Receptor for activated C kinase 1 promotes hepatocellular carcinoma growth by enhancing mitogen-activated protein kinase kinase 7 activity. Hepatology 57: 140-151, 2013.

12. Chou YC, Chou CC, Chen YK, et al: Structure and genomic organization of porcine RACK1 gene. Biochim Biophys Acta 1489: 315-322, 1999.

13. Lopez-Bergami P, Huang C, Goydos JS, et al: Rewired ERK-JNK signaling pathways in melanoma. Cancer Cell 11: 447-460, 2007.

14. Karin M: The regulation of AP-1 activity by mitogen-activated protein kinases. J Biol Chem 270: 16483-16486, 1995.

15. Zhang Y, Zhan Y, Zhang D, et al: Eupolyphaga sinensis walker displays inhibition on hepatocellular carcinoma through regulating cell growth and metastasis signaling. Sci Rep 4: 5518, 2014.

16. Choi DS, Young H, McMahon T, et al:The mouse RACK1 gene is regulated by nuclear factor-kappa $\mathrm{B}$ and contributes to cell survival. Mol Pharmacol 64: 1541-1548, 2003.

17. Jia D, Duan F, Peng P, et al: Up-regulation of RACK 1 by TGF- $\beta 1$ promotes hepatic fibrosis in mice. PLoS One 8: e60115, 2013. 